\title{
Research on Modified SVM for classification of SAR images
}

\author{
Peng Zhou ${ }^{1, a}$, Gang Guo ${ }^{2, b}$ and Fu Xiong ${ }^{3, c}$ \\ ${ }^{1}$ Huazhong University of Science and Technology, Wuhan 430074, China; \\ ${ }^{2}$ Shandong University, Jinan 250100, China. \\ ${ }^{3}$ Guangzhou University, Guangzhou 510300, China. \\ axsbandzjb@126.com, ${ }^{b} 554976360 @ q q . c o m,{ }^{c} 1361695520 @ q q . c o m$
}

Keywords: Synthetic radar images (SAR), SVM, Contourlet transform.

\begin{abstract}
Classification of synthetic radar images (SAR) is an emerging area especially with the advent of state of the art satellite image techniques. An SVM based texture analysis and classification utilizing the PCA for dimensionality reduction of SAR images has been presented in this paper to categorize the given SAR image into the water and urban areas. The experimentation has been conducted on 40 SAR images and the feature set size is 15. Finally, most effective 5 texture features are shortlisted for the classification of SAR images and accuracy is calculated by Specificity and Sensitivity test. The results obtained from test images give an accuracy of $94 \%$ for image classification. To make the algorithm adaptable, these textural features are reduced using principal component analysis (PCA), and principal components are used for classification purposes powered by a support vector machine classifier. The well known multiresolution approximation technique contourlet transform has been utilized in this paper to pre-process the input image in the frequency domain effectively and also to select the most significant features in the frequency domain. The proposed technique has been compared with conventional techniques such as the PCA and SVM in stand-alone approaches and the classification accuracy is increased along with the drastic reductions in the computation time.
\end{abstract}

\section{Introduction}

With the advent of satellites and technology, remote sensing plays a major role in the acquisition of data about an object or environmental phenomena without any physical contact or manual interventions. This provides a great advantage in regions which are uninhabitable or the terrain is unsuitable for human presence. A well-known recent development is the remote sensing data acquired from mars surface from the rover vehicle. Remote sensing also plays a major role in weather sensing and prediction. An important source of remote sensing is the use of synthetic aperture radar images (SAR) which form the source of remote sensing classification applications. SAR images give information about data which are collected from inaccessible areas and they include a wide area like information about ocean depths, the arctic and Antarctic glacial drift and breakdowns, weather currents, gulf streams etc., SAR images have the edge over their optical counterparts in the fact that they have good penetration abilities and can be used as an all-weather and terrain data acquisition method.

Considerable research contributions have been made in the past to implement classification algorithms on SAR images for a wide range of applications. Statistical feature extraction and classification methods have been applied to SAR target classification. Support Vector Machines (SVMs) are extensively used [2] and the accuracy analysis shows that SVMs perform more poorly than the Wishart classifier by approximately $16 \%$, whereas they perform better than the Maximum Likelihood, Minimum Distance, and Mahalanobis Distance classifiers by approximately 4\%, 17\% and $14 \%$, respectively. Moreover, the highest accuracy is achieved by using the coefficients of Krogager decomposition in the classification procedure. Feature extraction and classification in the frequency domain have been achieved with the help of wavelet transform and SVM for classification [17] where the field of classification is an oyster field cultivation and fusion rules used for classification. A fuzzy based classifier [1] where the features of the segmented area are extracted 
gives a high classification accuracy having an average of $92.56 \%$. The input test images were satellite images which were segmented using a modified graph cut algorithm. Texture measures are extracted using (a/the) statistical approaches like the discriminative power distance and PCA [5] used for classification of SAR images into rural and urban areas with a 95\&\% classification accuracy reported. Textural features have also been fused using principal component analysis (PCA), and principal components are used for classification purposes. To highlight the effectiveness of PCA, the difference between PCA- and non-PCA-based classifications is also analyzed [3].

In order to maximize the SAR classification accuracy, Binary Classifiers [18] has been implemented for optimal classifier architecture for each distinct terrain type and feature set whilst utilizing a large set of major features within. The CNBC further supports. The advantage of this method is that any terrain type can be classified using the developed framework but shows an increase in the computation time. Fusion based extraction and classification [16] have involved the use of registration procedures where two to four samples of the input images are registered against a standard reference image and feature level based fusion used for extracting the features.

Random [8] fields are frequently used to represent a variety of geophysical processes which develop in space and/or in time. This paper is devoted to the study of various aspects of multidimensional random fields by means of space transformations. These extracted features are given to a classifier to make the decisions. In general, discrete wavelets, Gabor wavelets, dual-tree complex wavelet are good extractors of textural information from the image under study. Hence, a good textural feature extractor combined with an optimum classifier forms the motivation behind this paper. A vast survey of SVM techniques have been observed in [11] where a divisive (top-down) approach is considered and set of classes are automatically separated into two smaller groups at each node of the hierarchy. The first algorithm splits the data samples based on a variation of the normalized cuts (NCuts) clustering algorithm wherein the weights of adjacency matrix are modified to utilize class membership in the process. The second algorithm also uses the NCuts clustering; however, it considers the involved classes rather than the individual data samples. It uses the minimum distances between the convex hulls of classes as a distance measure for determining the weights of the graph. Splits are determined for both algorithms based on the eigenvector corresponding to the second smallest eigenvalue of a Laplacian matrix, and it is observed that the proposed algorithms generate well-separated and well-balanced clusters. Unlike other clustering methods used for this purpose, the methods in the present study are found to be more suitable when SVMs are used as base classifiers.

The use of optimization methodologies [12] plays an important role in training SVMs. Due to different requirements on the training speed, memory constraint, and accuracy of optimization variables, practitioners should choose different optimization methods. It is thus instructive to review the optimization techniques used to train SVMs. Given that SVMs have been blended into the developments of other learning mechanisms, this would also be beneficial to facilitate readers to formulate and solve their own optimization objectives arising from SVM-related research.

This paper is organized as follows. Section 2 describes the proposed methodology. Section 3 illustrates the experimentation results and analysis followed by conclusion in section 4 .

\section{Proposed Work}

The proposed work utilizes a multiresolution approximation technique for extraction of features like variance, mean, etc., and providing the textural features to the PCA classifier to decide upon water and vegetative areas. The proposed workflow is shown in figure 1 where the input SAR image is pre pre-processed to remove speckle noise which is a form of multiplicative noise. Speckle noise is characteristic of the satellite as well as medical images. Feature selection is the important step in this block diagram. The CT estimated the feature from the input preprocessed image and then feature selection is based on the variable selection and subset selection. This technique is very useful for the redundant feature and the feature contains no information. It won't allow choosing the feature without information, which will not helpful for further processing. 


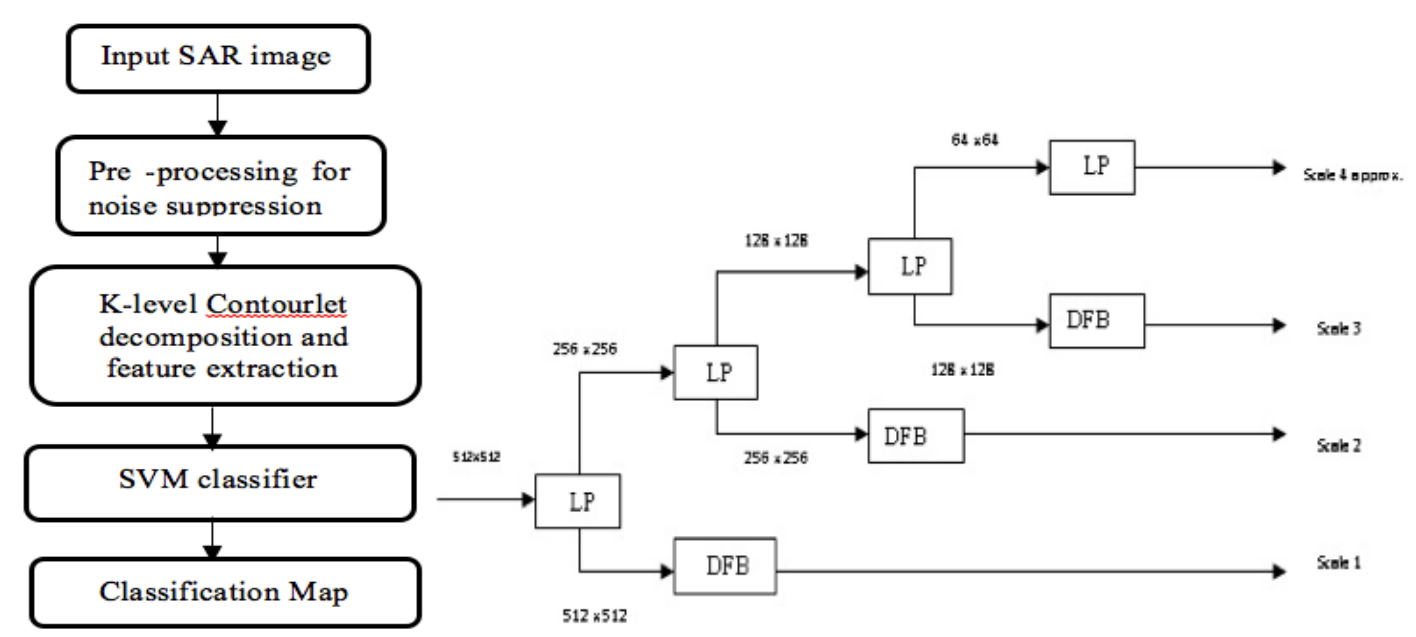

Fig. 1 Process flow proposed work

Fig. 2 Process flow proposed work

\subsection{The Contouret Transform}

The CT is a directional multiresolution image representation scheme proposed by Do and Vetterli, which is effective in representing smooth contours in different directions of an image, thus providing directionality and anisotropy [21]. The method utilizes a double filter bank, in order to obtain a sparse expansion of typical images containing smooth contours. In this filter bank shown in figure 2, first, the Laplacian Pyramid (LP) is used to detect the point discontinuities of the image and then a Directional Filter Bank (DFB) to link point discontinuities into linear structures. This scheme results in an image expansion that uses basic elements like contour segments and thus it is named Contourlet Transform. The Contourlet transform utilized in this paper which is a cascade of a Laplacian pyramid followed by a directional filter bank. Its structure is shown in figure 1 .

An essential feature of Contourlet transform is its high directionality and sparse representation [8] and its ability to identify and remove noise from high-frequency sub-bands. The Contourlet transform best approximates smooth discontinuities in a given image, unlike the Wavelet transform which is a good approximator of edges with sharp discontinuities. A 4 level decomposition of an input SAR image is shown in figure 3 .

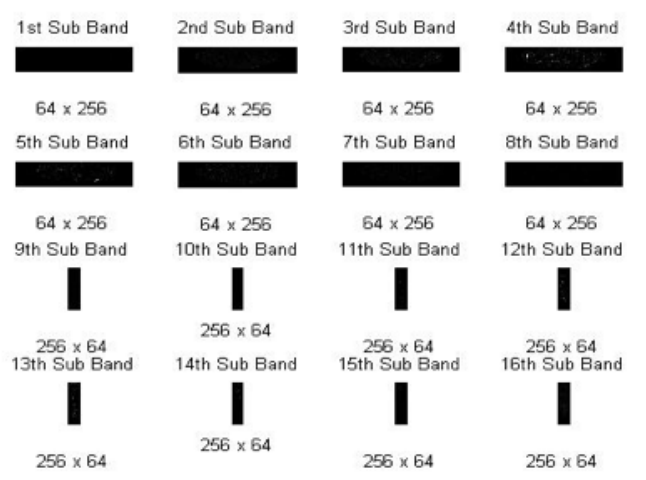

Fig. 34 level decomposition of input SAR image

Due to the iterated low-pass filtering, the most relevant texture information has been separated, thus the texture information is mainly contained in the directional sub-bands of each scale. As a result, the low-pass image is not taken into consideration when calculating the texture feature vector. A set of statistical texture features proposed in the literature [8][9] are evaluated in this study. Mean energy, standard deviation, and information entropy have been utilized for the Contourlet domain in [21]. The combination of an LP and DFB gives a double filter bank structure known as Contourlet filter bank. Band pass images from the LP are fed to DFB so that directional information can be captured. The scheme can be iterated the coarse image. This combination of LP and DFB stages result in a double iterated filter bank structure known as Contourlet filter bank. The Contourlet filter bank decomposes the given image into directional sub-bands at multiple scales. Figure 2 shows the decomposition of 
the image using Contourlet Transform for level-2 using 'pkva' filter for both low pass filter and direction filter bank.

The energy is one of the most effective features used in textural feature extraction. In this work, the mean energy of each subband image is calculated. Standard deviation provides a means to capture the scale of the diversity of the image whereas information entropy represents the complexity of the texture information. Contrast measures the amount of local variations present in the image, while homogeneity refers to the texture's contrast.

\subsection{Principal Component Analysis (PCA)}

The Principal component analysis extracts the principal components in an image and the multivalued and dimensional features are reduced to a single class. Hence they serve as good dimension reduction algorithms. The extracted features are represented as Eigenvalues. These Eigenvalues have a squared value equal to the addition of squared distances from their respective means. They are generally referred to as clouds and are orthonormal to each other. The first principal component corresponds to a line that passes through the multidimensional mean and minimizes the sum of squares of the distances of the points from the line. The second principal component corresponds to the same concept after all correlation with the first principal component has been subtracted from the points. Each value is proportional to the portion of the variance.

\subsection{SVM Classifier}

SVMs can be used to solve various real-world problems. Lots of work by using SVM in research has been done by the researchers. SVM is based on statistical learning theorem's VC Dimension concept and Structure Risk Minimization (SRM) principle thus generating an optimal separation hyper plane. The aim of SVM is to find best classification function to distinguish between members of the two classes in training data. For a linearly separable dataset, a linear classification function corresponds to a separating hyper plane $f(x)$ that passes through the middle of the two classes, separating of two. Once this function is determined, new data instance xn can be classified by simply testing the sign of the function

$$
f(x n): x n=+v e \text { if } f(x n)>0
$$

Because there are many such a linear hyper planes, what SVM additionally guarantee is that the best such a function is found by maximizing the margin between the two classes. Intuitively, the margin is defined as the amount of space or separation between the two classes as defined by hyper line. Geometrically, the margin corresponds to the shortest distance between the closest data points to a point on the hyper plane. SVM has two important features. Firstly, the upper bound on the generalization error does not depend on the dimension of the space. Secondly, the error bound is minimized by maximizing the margin, that is, the minimal distance between the hyper plane and the closest data points.can be used as a representation set of the whole big dataset.

\section{RESULTS AND DISCUSSION}

The input SAR image taken for experimentation is a CCD pattern image of Songpan Hengduan mountain ranges. The input image is subjected to pre-processing for removal of noise and features extracted through a $\mathrm{k}$ level Contourlet transform. The Contourlet transform as mentioned in the previous sections consists of a low pass visual component and a 16 high-frequency sub-band as the number of scale level has to be chosen to be at 4 . The SAR image is found to have different textures which are cropped to form 3-5 distinct textures per SAR image. The Contourlet transform is implemented using a Laplacian pyramid and Directional filter bank structure.
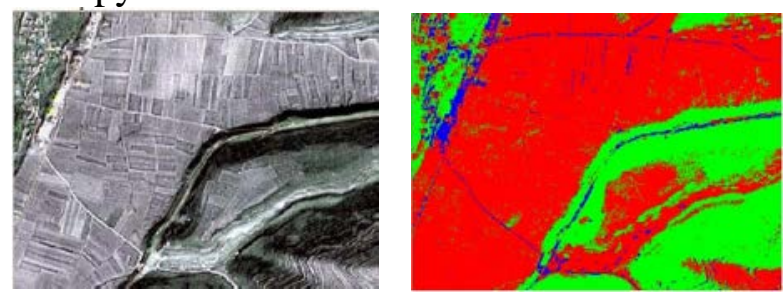

Fig. 4 Input SAR image and its classification amp 
To obtain a good quality feature set, the SAR images normalized and wiener filter used to remove the speckle noise. The input imageI(x,y) is decomposed into the $\mathrm{k}$ scales generating ${ }^{\wedge} \mathrm{ksub}$ bands and hence to construct the feature matrix. Each coefficient is having 64x64(total 2056) points. All the coefficients are arranged to make a column feature matrix $I^{\wedge} \mathrm{F}=\left\{\mathrm{I} \_1, \mathrm{I} \_2, \ldots . . \mathrm{I} \_\right.$nis constructed from the column vector and an image sub-space is created with the feature set. The weights are calculated for that particular image and stored in the vectorI $\wedge$ F. Euclidean distance is used as the parameter for comparison and the feature components are classified into water bodies or non - water bodies.

All the algorithms are implemented in MATLAB 7.0.1, Contourlet Toolbox and executed on the Pentium-I3,3.00GHz CPU with 4 GB RAM. For the Contourlet transform, pyramidal filter and directional filter the "pkva" filter is used. The two level subbands are used to find the coefficients. The image data compression by means of PC extraction is accomplished by projecting each sample block of the data along the directions of the individual orthonormal eigenvectors of the covariance matrix of the image data [7]. If the first few eigenvalues of the covariance matrix contain most of the signal energy, the dimensionality of the data can be greatly reduced without losing much information by only retaining those components along with the principal eigenvectors. In addition to its optimality in data reduction, which is not shared by other feature extraction schemes, it provides salient features of the data that are de-correlated. This property is ideally suited for classification purposes as these de-correlated features or components can be used to train the classifier effectively. As shown in the table the feature matrix for 33 images becomes 4096x 33=135168points. After applying PCA to the Feature Matrix I the weight matrix Tw becomes of size 33x33=1089 Points. Thus for the classification of the image required to match only 1089 points (33 x 33) and not 135168 points. Thus the computational cost greatly reduced by applying PCA.

Table 1 Performance evaluation of proposed work

\begin{tabular}{|l|l|l|l|}
\hline S.No. & Algorithm & Accuracy & Kappa \\
\hline 1. & Proposed work & $93.15 \%$ & 0.94 \\
\hline 2. & SVM & $91.79 \%$ & 0.89 \\
\hline 3. & PCA & $88.02 \%$ & 0.76 \\
\hline
\end{tabular}

Table 2 Computation time analysis

\begin{tabular}{|l|l|l|}
\hline S.No. & Algorithm & Computation time (s) \\
\hline 1. & Proposed work & 09.44 \\
\hline 2. & SVM & 11.97 \\
\hline 3. & PCA & 15.66 \\
\hline
\end{tabular}

The accuracy of a classification is assessed by comparing the classification results with the reference data, which has accurately reflected the types of ice cover in the SAR image. The accuracy of the classifier is measured by counting the number of pixels classified as the same in the satellite image and on the reference data, and then dividing these by the total number of pixels. The $\mathrm{k}$ coefficient is a measure of integrator agreement which is given as

$$
\mathrm{K}=\left(\mathrm{P} \_0-\mathrm{P} \_\mathrm{e}\right) /\left(1-\mathrm{P} \_\mathrm{e}\right)
$$

Kappa $\mathrm{K}$ is a positive value with its magnitude reflecting the strength of the integrator agreement and it becomes negative when the observed agreement is less than the chance agreement. To bring the best and suitable kernel for SVM, different kernels have been studied to find the reliable one. Table 1 describes the overall accuracy and $\mathrm{k}$-coefficient for each type of SVM kernels.

\section{Conclusion}

In order to evaluate the accuracy improvements in the classification of high-resolution imagery, the paper proposes a fusion and classification scheme of high resolution optical and SAR images. The Contourlet transform generates decomposition co-efficient(coefficient?) at a higher rate which is used to get new features from the input image and these features are helpful for better classification. The feature extraction using wavelet decomposition rate is less as against Contourlet transform. This novel technique not only gives significant results on the classification but most importantly, it extends 
the application of entropy decomposition to cover the multi-temporal data. Our future work will be done to evaluate on other useful parameters extracted from SAR data to further improve the classification accuracy The object-oriented information extraction approach can solve this problem. Fuzzy knowledge can play a more important role when high- resolution images are partitioned into objects for analysis. The experiments show that the use of fuzzy knowledge markedly improved the classification performance in comparison to traditional classification. This is explained by the fact that the fuzzy knowledge model built in experiments is based on spectral, spatial and texture attributes whose values do not significantly change over time. But the object-oriented method is not suited for all classes' classification. So how to combine the methods or find better approach are the key points in the future work. Maybe the multi-classifier combination can solve the problem.

\section{References}

[1] Yuhendra, Sumantyo, J.T.S., Kuze, H.: Performance Analyzing of High Resolution Pansharpening Techniques: Increasing Image Quality for Classification using Supervised Kernel Support Vector Machine. Research Journal of Information Technology 3(1), 12-23 (2011).

[2] R. Shah Hosseini, I. Entezari, S. Homayouni, M. Motagh \& B. Mansouri, "Classifcation of polarimetric SAR images using support vector machines”, Canadian Journal of Remote Sensing, Vol. 37, No. 2, 2011.

[3] Matken and Hajeb, "Oil Spill detection for SAR image using SVM based classification", International Archives of the Photogrammetry, Remote Sensing and Spatial Information Sciences, Volume XL-1/W3, 2013.

[4] YekkehKhany, Safari, Homayouni and Hasanlou, "A comparison study of different kernel functions for SVM based classification of multi temporal polarimetry SAR data ", The International Archives of the Photogrammetry, Remote Sensing and Spatial Information Sciences, Volume XL-2/W3, 2014

[5] Manjit Singh and Gunjit Kaur, "SAR image classification using PCA and texture analysis”, Information technology and Mobile Communication, pp. 435 - 439, 2011.

[6] Wei Gao, Jian Yang and Wenting Ma, "Land cover classification for polarimetric SAR images based on mixture models”, Journal of remote sensing, Vol. 6, No. 5, pp. 3770 - 3790, 2014.

[7] K. Kayabol and B. Gunsel, "Unsupervised classification of SAR images using normalized gamma process mixtures”, Digital Signal Processing, Vol. 23, No. 5, pp. 1344-1352, 2013.

[8] Xiuguo Liu1, Yongsheng Li , Wei Gao , Lin Xiao, “Double Polarization SAR Image Classification Based on Object-Oriented Technology, Journal of Geographic Information System, Vol. 2, pp. 113-119, 2010.

[9] Dae. Ki. Kang , Myoung-jong kim, Performance enhancement of SVM ensembles using genetic algorithms in Bankruptcy prediction , 2010 3rd international conference on advanced computer theory and engineering.

[10] P. Karunakar, V. Praveen and O. Ravi Kumar”Discrete Wavelet Transform-Based Satellite Image Resolution Enhancement” Advance in Electronic and Electric Engineering, Vol. 3, Number 4, pp. 405-412, 2013.

[11]H. Cevikalp, "New clustering algorithms for the support vector machine based hierarchical classification”, Pattern Recognition Letters, vol. 31, (2010), pp. 1285-1291.

[12] J. Taylor and S. Sun, “A review of optimization methodologies in support vector machines”, Neurocomputing, vol. 74, (2011), pp. 3609-3618. 
[13] Wang, Tan, Yang and Xuezhi”A multi-level SAR sea ice image classification method by incorporating egg-code-based expert knowledge” IEEE, 2012.

[14]Kanika Kalra, Anil Kumar Goswami and Rhythm Gupta "A Comparative Study of Supervised Image Classification Algorithms for Satellite Images" IJEEDC, 2013.

[15] Arindam Chaudhuri, Kajal De, Fuzzy support vector machine for bankruptcy prediction , 2011, applied soft computing 11(2011) 2472-2486.

[16]H. Skriver, F. Mattia, S. Member, G. Satalino, A. Balenzano, V. R. N. Pauwels, N. E. C. Verhoest, and M. Davidson, "Crop Classification Using Short-Revisit Multitemporal SAR Data," JSTARS, vol. 4, no. 2, pp. 423-431, 2011.

[17] O. Regniers, L. Bombrun and I. Ilea and V. Lafon, "Classification of oyster habitatsby combining wavelet based texture features and polarimetric SAR descriptors", proceedings of the international conference IEEE Geoscience and remote sensing. Pp. 3890 - 3890, 2015.

[18]G. Mountrakis, J. Im, and C. Ogole, "Support vector machines in remote sensing : A review," ISPRS J. Photogramm. Remote Sens., vol. 1, no. 315, pp. 1-13, 2010.

[19] Ching-Chang yeh, der -jang chi, Ming-fu hsu, A hybrid approach of DEA, rough set and support vector machine foe business failure prediction, Expert systems with applications, Vol. 37, 1535- 1541, 2010.

[20] Hui Li, Jie sun, "Predicting business failure using support vector machine with straight forward wrapper approach”, Expert system with applications, Vol. 38, pp. 12747-12756, 2011.

[21] Chitaliya \& Trivedi, “An Efficient Method for Face Feature Extraction and Recognition based on Contourlet Transform and Principal Component Analysis using Neural Network”, International Journal of Computer Applications (0975 - 8887) Volume 6- No.4, September 2010 